\title{
Factores sociodemográficos relacionados con el uso adecuado de las mascarillas y el distanciamiento social apropiado para evitar el contagio del SARS-CoV-2 en un mercado de abastos en Lima, Perú
}

Renzo Hurtado-Cuba* 1,a; Nora Espíritu 1,2,b

\section{RESUMEN}

Objetivo: Determinar la relación de los factores sociodemográficos con el uso adecuado de las mascarillas y el distanciamiento social apropiado para evitar el contagio del SARS-CoV-2 en las personas que visitan el mercado n. ${ }^{\circ} 2$ de Surquillo (Lima, Perú).

Materiales y métodos: Estudio cuantitativo, observacional, descriptivo, transversal y prospectivo comunitario. Se encuestó a 385 sujetos que acudieron al mercado de Surquillo entre septiembre y octubre del 2020 y se aplicó una lista de cotejo a los participantes. El tamaño muestral se obtuvo mediante EPIDAT 4.2 con una proporción esperada de prácticas inadecuadas del $50 \%$, nivel de confianza $95 \%$ y precisión absoluta del $5 \%$. El muestreo fue no probabilístico por conveniencia. La información fue analizada mediante SPSS v25. Se aplicó estadística descriptiva y analítica usando prueba de $\mathrm{X}^{2}$ y regresión logística, con un nivel de confianza del $95 \%$ y un $p<0,05$.

Resultados: El 48,80\% de los participantes fueron hombres. La edad promedio fue 42,61 $\pm 13,84$ años. El 64,70\% ( $n=249$ ) tuvo instrucción superior (técnico y universitario). El 47,50\% $(\mathrm{n}=183)$ de los encuestados procedía del distrito de Surquillo, y el $42,90 \%(n=165)$ fueron empleados independientes. El 48,80 \% ( $n=188)$ portaba una mascarilla en óptima condición. El $32,20 \%(n=124)$ cubría nariz y boca con un buen acople. La mascarilla de algodón fue la más usada: 35,10 \% de los participantes $(\mathrm{n}=135)$. El 70,00 \% opinó que el distanciamiento debe ser de 1,5 m. Durante la observación, el $94 \%$ mantuvo una distancia menor de 1,5 m. Existió una relación significativa entre el grado de instrucción superior y el uso adecuado de la mascarilla con $p<0,00$, pero no para el distanciamiento social.

Conclusiones: Existe una relación significativa entre el grado de instrucción y el uso adecuado de la mascarilla.

Palabras clave: COVID-19; SARS; Distancia social; Salud pública; Máscaras faciales; Pandemia (DeCS BIREME).

\section{Sociodemographic factors related to the proper use of masks and appropriate social distancing to prevent the spread of SARS-CoV-2 in a food market in Lima, Peru}

\author{
ABSTRACT
}

Objective: To determine the relationship that sociodemographic factors have with the proper use of masks and appropriate social distancing to prevent the spread of SARS-CoV-2 in people who attend the Surquillo No. 2 market (Lima, Peru). Materials and methods: A quantitative, observational, descriptive, cross-sectional and prospective community study. Both a survey and a checklist were administered to 385 subjects who attended the Surquillo market between September and October 2020. The sample size was obtained using Epidat 4.2 with an expected proportion of inadequate practices of $50 \%$, a confidence level of $95 \%$ and an absolute precision of $5 \%$. Non-probability convenience sampling was used. The information was analyzed using IBM SPSS Statistics V25. Descriptive and analytical statistics were performed using the chi-square test and logistic regression with a confidence level of $95 \%$ and $p<0.05$.

Results: Forty-eight point eight zero percent (48.80\%) of the research participants were males. Their mean age was $42.61 \pm 13.84$ years. Sixty-four point seven zero percent $(64.70 \%)(n=249)$ had higher (technical and university) education, $47.50 \%(n=183)$ lived in Surquillo, $42.90 \%(n=165)$ were self-employed, $48.80 \%(n=188)$ wore a well-fitting mask in good condition, $32.20 \%(n=124)$ covered their nose and mouth properly, $35.10 \%(n=135)$ used cotton masks, $70.00 \%$ thought that a distance of $1.5 \mathrm{~m}$ should be kept from others, and $94 \%$ maintained a physical distance of less than $1.5 \mathrm{~m}$ during the observation. There was a significant relationship between the higher education level and the proper use of masks $(p<0.001)$ but not between the education level and social distancing.

Conclusions: There is a significant relationship between the education level and the proper use of masks.

Keywords: Coronavirus infections; Severe acute respiratory syndrome; Social distance; Public health; Facial masks; Pandemics (Source: MeSH NLM).

1 Universidad de San Martin de Porres, Facultad de Medicina Humana. Lima, Perú.

2 Hospital Nacional Dos de Mayo. Lima, Perú.

a Alumno de Medicina Humana.

b Médica Pediatra, Magíster en Salud Pública.

*Autor corresponsal. 


\section{INTRODUCCIÓN}

Desde inicios del 2020 la población mundial se ha visto afectada por una pandemia que ha revelado las debilidades y fortalezas de nuestros sistemas de salud, políticos y sociales. Junto con esta situación, se han fomentado una serie de medidas sanitarias para evitar el contagio por el SARS-CoV-2 en distintos espacios públicos como bancos, mercados, entidades públicas, servicios de transporte masivo, entre otros.

En el continente americano, el uso de las mascarillas faciales por la comunidad ha sido variable, a diferencia de los países asiáticos donde se emplean con más frecuencia, probablemente por la experiencia adquirida desde la epidemia del coronavirus SARS en el año 2003 y otras anteriores. Se ha demostrado que el uso de mascarillas, la desinfección de los ambientes y el lavado de manos fueron factores protectores efectivos para limitar la transmisión de la enfermedad (1). Durante los primeros cien días del brote de coronavirus, en marzo de 2020, en Hong Kong se evaluó el cumplimiento del uso de las mascarillas, que alcanzó el $96 \%$ y tuvo una incidencia en el contagio de 129 infectados por millón de habitantes: se concluyó que el uso adecuado y masivo de las mascarillas contribuye al control de la COVID-19, ya que reduce la cantidad de emisiones de saliva y microgotas respiratorias de los individuos portadores de la enfermedad (2). Asimismo, se enfatiza mantener un distanciamiento social y una adecuada ventilación en espacios cerrados para evitar nuevos contagios de COVID-19. Un estudio indica que el distanciamiento social es seguro entre 1,60 y 3 metros cuando se considera la transmisión por gotas grandes exhaladas al hablar ${ }^{(3)}$.

El último reporte situacional de la Organización Mundial de la Salud (OMS), emitido el primero de junio del 2021, muestra una disminución de los nuevos casos y fallecidos en todo el mundo. Solo en la última semana de junio se contabilizaron 3,5 millones de nuevos casos y 78000 nuevos fallecidos, lo que refleja una disminución porcentual del $15 \%$ y 7 \%, respectivamente. En América, se observa una discreta caída en los nuevos casos y los fallecidos, en comparación con el reporte semanal anterior. Estos números parecen alentadores, pero las medidas de protección personal no deben descuidarse, ya que, al tratarse de una enfermedad relativamente nueva, no se sabe aún si existirá una nueva ola de infectados. Asimismo, se resalta que la incidencia de casos y muertes permanece elevada, y además, se ha reportado un incremento de la incidencia en algunos países ${ }^{(4)}$.

En el Perú, según la información de la plataforma digital única del Estado peruano, se han realizado más de 12839000 pruebas, de las cuales 1955469 resultaron positivas $(15,23 \%$ de positividad). Así también, se indica que existen más de 1897000 personas recuperadas y 69342 fallecidos, lo que representa una mortalidad del $3,55 \%$ (5).
Tras una actualización emitida en la última semana de mayo con respecto al balance oficial de fallecidos por la pandemia, la situación nos coloca como el país con la mayor tasa de mortalidad del mundo con 5484 fallecidos por millón de habitantes ${ }^{(6)}$. Se ha pasado de 69342 a 180764 fallecidos, luego de una variación de los criterios de consideración para los fallecidos durante la pandemia ${ }^{(7)}$.

La campaña de vacunación en el Perú se inició en febrero. Al primero de junio del 2021, se han aplicado 4140000 dosis, con un total de personas vacunadas de 1253239 que ya recibieron las dos dosis ${ }^{(8)}$.

Ante esta situación crítica como país, se planteó esta investigación con el objetivo de determinar la relación de los factores sociodemográficos con el uso adecuado de mascarillas y el distanciamiento social apropiado para evitar el contagio del SARS-CoV-2 en la población que visita el mercado de abastos $n .^{\circ} 2$ del distrito de Surquillo en la ciudad de Lima, en el periodo septiembre a octubre del año 2020.

\section{MATERIALES Y METODOS}

\section{Diseño y población de estudio}

Estudio cuantitativo, observacional, descriptivo, transversal y prospectivo comunitario. La población del estudio estuvo constituida por las personas de la comunidad que acuden al mercado $\mathrm{n} .{ }^{\circ} 2$ del distrito de Surquillo en Lima, Perú.

El mercado $n .{ }^{\circ} 2$ de Surquillo es un centro de abastos que, para su reapertura, debió cumplir con el protocolo de seguridad establecido por el municipio (toma de temperatura en los ingresos, verificación del uso de mascarillas a todo el que ingresa, aplicación de alcohol en gel a las manos de los visitantes y pruebas periódicas a los trabajadores del mercado). El establecimiento se ubica en el jirón Leonardo Barbieri 750 del distrito de Surquillo $\mathrm{y}$ ofrece productos de primera necesidad como frutas, verduras, carnes y artículos no perecibles, artículos de limpieza, artículos de bazar, etc.

\section{Variables y mediciones}

Un promedio de 5000 personas acude diariamente al mercado de abastos $\mathrm{n} .{ }^{\circ} 2$ de Surquillo. La muestra fue de 385 sujetos y fue obtenida mediante EPIDAT versión 4.2, se asumió una proporción esperada del $50 \%$ de malas prácticas, un nivel de confianza del $95 \%$ y una precisión del $5 \%$. El muestreo fue no probabilístico, por conveniencia. Se encuestó a las personas que acudieron al mercado entre septiembre y octubre del 2020, hasta completar el tamaño muestral. En el estudio fueron incluidos los mayores de 18 años, en pleno uso de sus facultades mentales y que dieron su consentimiento informado para responder la 
Factores sociodemográficos relacionados con el uso adecuado de las mascarillas y el distanciamiento social apropiado para evitar el contagio del SARS-CoV-2 en un mercado de abastos en Lima, Perú

encuesta. Se excluyeron a las personas con dificultad para comunicarse o que se negaron a responder la encuesta. Los entrevistadores fueron capacitados en el reconocimiento del uso adecuado de las mascarillas (2) y en la medición del distanciamiento social ${ }^{(3)}$. En todo momento se usaron los equipos de protección personal.

Se elaboró un instrumento de recolección de datos tipo encuesta de siete preguntas de respuestas múltiples. Las variables que se recogieron fueron edad, sexo, grado de instrucción, distrito de procedencia, situación laboral, frecuencia del uso de las mascarillas, opinión sobre el distanciamiento social, información sobre pruebas de despistaje e infección por SARS-CoV-2 en el entorno familiar inmediato y lejano. Mediante una lista de cotejo de cuatro puntos, se observó el tipo de material, condiciones y cómo estaban usando las mascarillas en el momento de la encuesta, si usaban el protector facial y se valoró el distanciamiento social mediante el uso de una cinta métrica, para lo cual se capacitó previamente a las personas que lo aplicarían. El uso de las mascarillas fue calificado como adecuado si se cumplían tres condiciones: mascarilla de tipo correcto (quirúrgica de 3 pliegues, respirador N95, respirador FFP1, FFP2, FFP3 o respirador N95 con válvula exhaladora), condición de la mascarilla (física, desgaste por el uso) y si cubría adecuadamente boca y nariz con buen acople. El distanciamiento social adecuado se consideró como 1,50 metros o más ${ }^{(3)}$. Ambos instrumentos fueron validados por 7 médicos: 2 infectólogos, 3 epidemiólogos y 2 investigadores, cuyas recomendaciones contribuyeron a su mejora.

\section{Análisis estadístico}

La información recolectada fue analizada y tabulada con el programa SPSS versión 25 . Se realizó un análisis univariado mediante la estadística descriptiva. Las variables cualitativas se expresaron en frecuencias absolutas y relativas; y las variables cuantitativas, en medidas de tendencia central y desviación estándar. El análisis bivariado se realizó por asociación de las variables sociodemográficas con el uso adecuado de la mascarilla y con el distanciamiento social mediante la prueba de $\mathrm{X}^{2}$ (chi cuadrado). Los factores en los cuales se encontró un valor $p$ significativo $(<0,05)$ fueron ingresados a un modelo de regresión logística.

\section{Consideraciones éticas}

El protocolo fue revisado y aprobado por el Comité de Ética de la Facultad de Medicina Humana de la Universidad de San Martin de Porres. Se solicitó el consentimiento informado a los sujetos antes de su participación. La encuesta fue anónima. Este trabajo siguió las normas de buena práctica clínica y en todo momento cumplió con las normas éticas.

\section{RESULTADOS}

La tabla 1 muestra las características sociodemográficas de los 385 participantes. De ellos, 88 (48,80\%) fueron hombres y 197 $(51,20 \%)$, mujeres. La edad promedio fue $42,61 \pm 13,84$, y el rango de edad predominante fue de 31 a 45 años, con un $36,36 \%(n=140)$. En cuanto al grado de instrucción, se encontró que un $64,67 \%(n=249)$ tenía instrucción superior (incluye técnico y universitario); el distrito de procedencia más frecuente fue Surquillo, con 47,54 \% ( $n=183)$; y el $42,90 \%(n=165)$ eran trabajadores independientes.

Tabla 1. Características sociodemográficas de las personas que acuden al mercado de abastos n. ${ }^{\circ} 2$ de Surquillo. Setiembre-octubre 2020. Lima, Perú

\begin{tabular}{|c|c|c|}
\hline \multirow{2}{*}{$\begin{array}{l}\text { Caracteristicas } \\
\text { sociodemográficas }\end{array}$} & \multicolumn{2}{|c|}{$\mathrm{n}=385$} \\
\hline & $\mathrm{n}$ & $\%$ \\
\hline Edad (media \pm DS) & $42,61 \pm 13,84$ & \\
\hline \multicolumn{3}{|l|}{ Edad (años) } \\
\hline $18-30$ & 88 & 22,86 \\
\hline $31-45$ & 140 & 36,36 \\
\hline $46-60$ & 104 & 27,01 \\
\hline$>60$ & 53 & 13,77 \\
\hline \multicolumn{3}{|l|}{ Sexo } \\
\hline Masculino & 188 & 48,80 \\
\hline Femenino & 197 & 51,20 \\
\hline \multicolumn{3}{|l|}{ Grado de instrucción } \\
\hline Primaria & 11 & 2,86 \\
\hline Secundaria & 125 & 32,47 \\
\hline Técnico & 127 & 32,98 \\
\hline Universitario & 122 & 31,69 \\
\hline
\end{tabular}




\begin{tabular}{|lcr|}
\hline \multicolumn{1}{c}{ Características } & \multicolumn{2}{c}{$n=385$} \\
\hline \multicolumn{1}{c}{ sociodemográficas } & $n$ & \\
\hline Distrito de procedencia & 183 & 47,54 \\
\hline Surquillo & 53 & 13,77 \\
\hline Miraflores & 28 & 7,27 \\
\hline San Borja & 32 & 8,31 \\
\hline Surco & 8 & 2,07 \\
\hline Barranco & 10 & 2,59 \\
\hline Chorrillos & 71 & 18,45 \\
\hline Otros & & \\
\hline Ocupación & 44 & 11,40 \\
\hline Desempleado & 117 & 30,40 \\
\hline Empleado dependiente & 165 & 42,90 \\
\hline Empleado independiente & 59 & 15,30 \\
\hline Otros (estudiante, ama de casa) & & \\
\hline
\end{tabular}

Respecto al uso de las mascarillas, el 99,20\% ( $\mathrm{n}=382$ ) refieren que siempre usan las mascarillas al salir de su casa. El tipo de mascarilla más utilizada fue la artesanal de tela de algodón con un 35,10\% $(n=135)$; en cuanto a las condiciones de las mascarillas el 48,83\% $(n=188)$ estaban en óptimas condiciones, y el $48,31 \%(n=186)$ con un desgaste propio del uso, sin compromiso de su funcionalidad. La mascarilla cubría nariz y boca con un buen acople en el 32,20\% $(n=124)$ de las personas (Tabla 2$)$.

Tabla 2. Características reportadas y observadas en el uso de las mascarillas por las personas que acuden al mercado de abastos n. ${ }^{\circ} 2$ del distrito de Surquillo. Setiembre-octubre 2020. Lima, Perú

\begin{tabular}{|lrr|}
\hline \multicolumn{1}{|c|}{$\begin{array}{c}\text { Caracteristicas uso } \\
\text { de mascarillas }\end{array}$} & \multicolumn{2}{c|}{$n$} \\
\hline Frecuencia reportada del uso de las mascarillas & \\
\hline Siempre & 382 & 99,20 \\
\hline Casi siempre & 3 & 0,80 \\
\hline A veces & 0 & 0,00 \\
\hline Rara vez & 0 & 0,00 \\
\hline Nunca & 0 & 0,00 \\
\hline Tipo de mascarilla observada & & \\
\hline Quirúrgica (3 pliegues) & 121 & 31,40 \\
\hline N95 (FFP1) & 75 & 19,50 \\
\hline N95 (FFP2/FFP3) & 25 & 6,50 \\
\hline N95 con válvula exhaladora & 9 & 2,30 \\
\hline Artesanal (1 capa) & 135 & 35,10 \\
\hline Neopreno & 20 & 5,20 \\
\hline Condiciones observadas de la mascarilla & & \\
\hline Óptima & 188 & 48,83 \\
\hline Desgaste propio del uso & 186 & 48,31 \\
\hline Con rasgaduras & 5 & 1,30 \\
\hline Con agujeros & 2 & 0,52 \\
\hline Con agujeros y rasgaduras & 2 & 0,52 \\
\hline Tiras de ajuste rotas & 2 & 0,52 \\
\hline
\end{tabular}


Factores sociodemográficos relacionados con el uso adecuado de las mascarillas y el distanciamiento social apropiado para evitar el contagio del SARS-CoV-2 en un mercado de abastos en Lima, Perú

\begin{tabular}{|c|c|c|}
\hline \multirow{2}{*}{$\begin{array}{l}\text { Características uso } \\
\text { de mascarillas }\end{array}$} & \multicolumn{2}{|c|}{$n=385$} \\
\hline & $\mathrm{n}$ & $\%$ \\
\hline \multicolumn{3}{|l|}{ Uso de la mascarilla } \\
\hline No lleva & 0 & 0,00 \\
\hline No cubre nariz ni boca & 5 & 1,30 \\
\hline Cubre solo boca & 42 & 10,90 \\
\hline Cubre nariz y boca con buen acople & 124 & 32,20 \\
\hline Cubre nariz y boca sin acople & 214 & 55,60 \\
\hline
\end{tabular}

La tabla 3 muestra los resultados en relación con el distanciamiento social: casi el 70,00 \% opinó que el distanciamiento debe de ser de 1,50 m a más; sin embargo, en la medición realizada, el 94,00 \% mantuvo una distancia menor de 1,50 m.

Tabla 3. Características sobre el distanciamiento social por las personas que acuden al mercado de abastos $\mathrm{n} .{ }^{\circ} 2$ del distrito de Surquillo. Setiembre-octubre 2020. Lima, Perú

\begin{tabular}{|c|c|c|}
\hline \multirow{2}{*}{$\begin{array}{c}\text { Distanciamiento } \\
\text { social }\end{array}$} & \multicolumn{2}{|c|}{$\mathrm{n}=385$} \\
\hline & $\mathrm{n}$ & $\%$ \\
\hline \multicolumn{3}{|c|}{ Opinión sobre distanciamiento adecuado } \\
\hline $0,50 \mathrm{~m}$ & 0 & 0,00 \\
\hline $1 \mathrm{~m}$ & 93 & 24,15 \\
\hline $1,50 \mathrm{~m}$ & 190 & 49,35 \\
\hline $2 \mathrm{~m}$ & 87 & 22,60 \\
\hline$>2 \mathrm{~m}$ & 15 & 3,90 \\
\hline No es necesario & 0 & 0,00 \\
\hline \multicolumn{3}{|c|}{ Cuantificación observada del distanciamiento } \\
\hline$>2 \mathrm{~m}$ & 1 & 0,30 \\
\hline$>1,50 \mathrm{~m} /<2 \mathrm{~m}$ & 22 & 5,70 \\
\hline$>1 \mathrm{~m} /<1,50 \mathrm{~m}$ & 209 & 54,30 \\
\hline$<1 \mathrm{~m}$ & 153 & 39,70 \\
\hline
\end{tabular}

En la tabla 4 se observa que el 50,64\% ( $n=195)$ de los participantes no se había realizado ninguna prueba para diagnóstico de COVID-19; el 40,52 \% se realizó alguna prueba con resultado negativo, y solo un $8,84 \%$ se realizó una prueba cuyo resultado fue positivo (porcentaje de positividad de $17,89 \%)$. El tipo de examen más frecuente fue la prueba rápida con un $42,90 \%(n=165)$, y el $3,90 \%$ se realizó la prueba molecular.
En cuanto al entorno familiar de los participantes con COVID-19, el $82,10 \%(n=316)$ reportó no haber tenido algún familiar enfermo en el ámbito hogareño inmediato; sin embargo, el $68,10 \%(n=262)$ refirió que algún familiar contrajo la enfermedad, pero que no vivía bajo el mismo techo (Tabla 4). 
Tabla 4. Pruebas covid19 realizadas por personas que acuden al mercado de abastos y casos COVID-19 dentro y fuera de su entorno familiar. Setiembre-octubre 2020

\begin{tabular}{|crr|}
\hline & \multicolumn{2}{c}{$n=385$} \\
\cline { 2 - 3 } Pruebas y casos CoVID-19 & $n$ & \\
& & \\
\hline Resultados de las pruebas realizadas & 34 & 8,84 \\
\hline Positivo & 156 & 40,52 \\
\hline Negativo & 195 & 50,64 \\
\hline No se ha realizado & & \\
\hline Tipo de pruebas realizadas & 165 & 42,90 \\
\hline Prueba rápida & 15 & 3,90 \\
\hline Prueba molecular & 9 & 2,30 \\
\hline Ambas & 196 & 50,90 \\
\hline No se ha realizado & & \\
\hline COVID-19 en casa & 69 & 17,90 \\
\hline Positivo & 316 & 82,10 \\
\hline Negativo & & \\
\hline COVID-19 fuera de casa (familiar) & 262 & 68,10 \\
\hline Positivo & 123 & 31,90 \\
\hline Negativo & & \\
\hline
\end{tabular}

El $27,80 \%(n=107)$ cumplió con el uso adecuado de las mascarillas (tipo, condiciones óptimas y cubre boca y nariz con buen acople), mientras que el $72,20 \%(n=278)$ participantes no lo hizo. En el análisis bivariado, algunos factores sociodemográficos estuvieron relacionados con el uso adecuado de las mascarillas (Tabla 5). Se encontró entre los participantes con grado de instrucción superior una relación significativa con el uso adecuado de las mascarillas $(p<0,001)$; así como en los participantes del distrito de Miraflores y de ocupación empleado $(p<0,005)$.

Tabla 5. Factores sociodemográficos relacionados con el uso adecuado/no adecuado de las mascarillas. Setiembre-octubre 2020.Lima, Perú

\begin{tabular}{|c|c|c|c|c|c|c|}
\hline \multirow{2}{*}{$\begin{array}{l}\text { Características } \\
\text { sociodemográficas }\end{array}$} & \multicolumn{2}{|c|}{$\begin{array}{c}\text { Uso adecuado } \\
n=107 \\
\end{array}$} & \multicolumn{2}{|c|}{$\begin{array}{l}\text { Uso no adecuado } \\
n=278 \\
\end{array}$} & \multirow[t]{2}{*}{$x^{2}$} & \multirow[t]{2}{*}{$p$} \\
\hline & $\mathrm{n}$ & $\%$ & $\mathrm{n}$ & $\%$ & & \\
\hline \multicolumn{7}{|l|}{ Edad } \\
\hline$<45$ años & 61 & 57 & 167 & 60,10 & 0,300 & $>0,05$ \\
\hline$>45$ años & 46 & 43 & 111 & 39,90 & & \\
\hline \multicolumn{7}{|l|}{ Sexo } \\
\hline Masculino & 49 & 45,80 & 139 & 50 & 0,547 & $>0,05$ \\
\hline Femenino & 58 & 54,20 & 139 & 50 & & \\
\hline \multicolumn{7}{|l|}{ Grado de instrucción } \\
\hline Básica & 23 & 21,50 & 113 & 40,60 & 12,405 & $<0,001$ \\
\hline Superior & 84 & 78,50 & 165 & 59,40 & & \\
\hline \multicolumn{7}{|l|}{ Distrito de procedencia } \\
\hline Surquillo & 39 & 36,40 & 144 & 51,80 & & \\
\hline Miraflores & 22 & 20,60 & 31 & 11,15 & 9,504 & $<0,05$ \\
\hline Otros & 46 & 43 & 103 & 37,05 & & \\
\hline \multicolumn{7}{|l|}{ Ocupación } \\
\hline Desempleado & 8 & 7,50 & 36 & 12,90 & & \\
\hline Empleado & 88 & 82,20 & 194 & 69,80 & 6,123 & $<0,05$ \\
\hline Otros & 11 & 10,30 & 48 & 17,30 & & \\
\hline
\end{tabular}


Factores sociodemográficos relacionados con el uso adecuado de las mascarillas y el distanciamiento social apropiado para evitar el contagio del SARS-CoV-2 en un mercado de abastos en Lima, Perú

En el análisis bivariado entre los factores sociodemográficos y el distanciamiento social adecuado, no se encontró relación significativa (Tabla 6).

Tabla 6. Factores sociodemográficos relacionados con el distanciamiento social apropiado por las personas que acuden al mercado de abastos n. ${ }^{\circ} 2$ de Surquillo. Setiembre-octubre 2020. Lima, Perú

\begin{tabular}{|c|c|c|c|c|c|c|}
\hline \multirow{2}{*}{$\begin{array}{c}\text { Características } \\
\text { sociodemográficas }\end{array}$} & \multicolumn{2}{|c|}{$\begin{array}{c}\text { Adecuado } \\
n=23\end{array}$} & \multicolumn{2}{|c|}{$\begin{array}{c}\text { No adecuado } \\
n=362\end{array}$} & \multirow[t]{2}{*}{$x^{2}$} & \multirow[t]{2}{*}{$p$} \\
\hline & $\mathrm{n}$ & $\%$ & $\mathrm{n}$ & $\%$ & & \\
\hline \multicolumn{7}{|l|}{ Edad en 2 categorías } \\
\hline$<45$ años & 13 & $(56,5)$ & 215 & $(59,4)$ & 0,074 & $>0,05$ \\
\hline >45 años & 10 & $(43,5)$ & 147 & $(40,6)$ & & \\
\hline \multicolumn{7}{|l|}{ Sexo } \\
\hline Masculino & 10 & $(43,5)$ & 178 & $(49,2)$ & 0,281 & $>0,05$ \\
\hline Femenino & 13 & $(56,5)$ & 184 & $(50,8)$ & & \\
\hline \multicolumn{7}{|l|}{ Grado de instrucción } \\
\hline Básica & 6 & $(26,1)$ & 130 & $(35,9)$ & 0,914 & $>0,05$ \\
\hline Superior & 17 & $(73,9)$ & 232 & $(64,1)$ & & \\
\hline \multicolumn{7}{|l|}{ Distrito de procedencia } \\
\hline Surquillo & 8 & $(34,8)$ & 175 & $(48,3)$ & & \\
\hline Miraflores & 4 & $(17,4)$ & 49 & $(13,5)$ & 1,596 & $>0,05$ \\
\hline Otros & 11 & $(47,8)$ & 138 & $(38,1)$ & & \\
\hline \multicolumn{7}{|l|}{ Ocupación } \\
\hline Desempleado & 1 & $(4,3)$ & 43 & $(11,9)$ & & \\
\hline Empleado & 18 & $(78,3)$ & 264 & $(72,9)$ & 1,225 & $>0,05$ \\
\hline Otro & 4 & $(17,4)$ & 55 & $(15,2)$ & & \\
\hline
\end{tabular}

En la tabla 7 se muestran los resultados de la regresión logística de los factores sociodemográficos con el uso adecuado de las mascarillas, en los que es significativo el grado de instrucción, con un valor de $p<0,001$.

Tabla 7. Análisis multivariado de los factores asociados al uso adecuado de las mascarillas por las personas que acuden al mercado de abastos n. ${ }^{\circ} 2$ de Surquillo. Setiembre-octubre 2020

\begin{tabular}{|c|c|c|c|c|c|c|c|}
\hline & \multirow{2}{*}{ B } & \multirow{2}{*}{$\begin{array}{l}\text { Error } \\
\text { estándar }\end{array}$} & \multirow{2}{*}{ gl } & \multirow{2}{*}{ Sig. } & \multirow{2}{*}{ OR } & \multicolumn{2}{|c|}{$95 \%$ C.I. para EXP(B) } \\
\hline & & & & & & Inferior & Superior \\
\hline Edad $(<45,>45)$ & 0,311 & 0,244 & 1 & 0,202 & 1,365 & 0,847 & 2,200 \\
\hline Sexo & 0,279 & 0,244 & 1 & 0,253 & 1,322 & 0,819 & 2,133 \\
\hline Grado de instrucción en 2 categorías & 0,868 & 0,270 & 1 & 0,001 & 2,383 & 1,404 & 4,046 \\
\hline Distrito de procedencia en 3 categorías & 0,155 & 0,130 & 1 & 0,232 & 1,168 & 0,905 & 1,506 \\
\hline Ocupación en 3 categorías & & & 2 & 0,059 & & & \\
\hline Ocupación en 3 categorías (1) & 0,781 & 0,428 & 1 & 0,068 & 2,184 & 0,943 & 5,054 \\
\hline Ocupación en 3 categorías (2) & 0,094 & 0,531 & 1 & 0,860 & 1,098 & 0,387 & 3,112 \\
\hline Constante & $-4,205$ & 0,875 & 1 & 0,000 & 0,015 & & \\
\hline
\end{tabular}

a. Variables incluidas en el modelo de regresión logística 1: edad $>45<$, sexo (M/F), grado de instrucción (básico, superior), distrito de procedencia (Surquillo, Miraflores, otros). 
La tabla 8 evidencia que no existe relación significativa entre la regresión logística de los factores sociodemográficos con el distanciamiento social apropiado (valores $p>0,05$ ).

Tabla 8. Análisis multivariado de los factores asociados al uso distanciamiento social apropiado por las personas que acuden al mercado de abastos $n .{ }^{\circ} 2$ de Surquillo. Setiembre-octubre 2020

\begin{tabular}{|c|c|c|c|c|c|c|c|}
\hline & \multirow[t]{2}{*}{ B } & \multirow{2}{*}{$\begin{array}{c}\text { Error } \\
\text { estándar }\end{array}$} & \multirow{2}{*}{$\mathrm{gl}$} & \multirow{2}{*}{ Sig. } & \multirow{2}{*}{ OR } & \multicolumn{2}{|c|}{$95 \%$ C.I. para EXP(B) } \\
\hline & & & & & & Inferior & Superior \\
\hline Edad dicotomizada & 0,222 & 0,446 & 1 & 0,618 & 1,249 & 0,521 & 2,992 \\
\hline Sexo & 0,200 & 0,458 & 1 & 0,663 & 1,221 & 0,497 & 3,000 \\
\hline Grado de instrucción en 2 categorías & 0,427 & 0,495 & 1 & 0,389 & 1,532 & 0,581 & 4,043 \\
\hline Distrito de procedencia en 3 categorías & 0,240 & 0,242 & 1 & 0,323 & 1,271 & 0,790 & 2,043 \\
\hline Ocupación en 3 categorías & & & 2 & 0,572 & & & \\
\hline Ocupación en 3 categorías (1) & 1,062 & 1,055 & 1 & 0,314 & 2,893 & 0,366 & 22,892 \\
\hline Ocupación en 3 categorías ( 2 ) & 1,179 & 1,153 & 1 & 0,306 & 3,253 & 0,340 & 31,142 \\
\hline Constante & $-5,585$ & 1,732 & 1 & 0,001 & 0,004 & & \\
\hline
\end{tabular}

b. Variables especificadas en el paso 1: Edad dicotomizada, sexo, grado de instrucción en 2 categorías, distrito de procedencia en 3 categorías, ocupación en 3 categorías.

\section{DISCUSIÓN}

Los resultados de esta investigación muestran que existe una relación significativa entre el grado de instrucción con el uso adecuado de las mascarillas por las personas que acuden al mercado de abastos $n .^{\circ} 2$ del distrito de Surquillo.

Si bien el uso de mascarillas es mandatorio en la actualidad, no hay indicaciones sobre qué tipo de mascarillas son más adecuadas para la prevención de un contagio ${ }^{(9)}$. El 35,10\% de los participantes en este estudio usó mascarillas artesanales de tela de algodón de una sola capa, que no son las adecuadas, según Fisher et al., ya que ofrecen una protección inferior en comparación con las demás mascarillas disponibles ${ }^{(3)}$. Al inicio de la pandemia, Greenhalgh et al. señalaron que el "principio de precaución", definido como el uso de medidas no farmacológicas como el uso de mascarillas artesanales de algodón o caseras, es un mecanismo válido para la prevención, a pesar de ser menos eficaces que las mascarillas N95 para disminuir el contacto con las microgotas o secreciones producidas por posibles personas infectadas en el entorno inmediato ${ }^{(10)}$.

Gandhi et al. sostienen que el uso universal de las mascarillas disminuiría el inóculo de partículas cargadas con el SARS-CoV-2, y ayudaría a reducir la gravedad de la enfermedad y a que una proporción de nuevas infecciones cursen como asintomáticas, lo que resulta en un estímulo que generaría inmunidad y, por lo tanto, disminuiría la propagación del virus mientras se espera la llegada de la vacuna (11). Li et al., combinando modelos matemáticos y evidencia científica existente, concluyeron que las mascarillas faciales cumplen al menos con dos funciones importantes. La primera es disminuir la formación de una nube de gas turbulento que se produciría después de toser o estornudar; y la segunda, una disminución de la emisión de patógenos respiratorios. Además, afirman que una persona infectada y asintomática que usa mascarillas puede reducir potencialmente el riesgo de infectar a otra persona ${ }^{(12)}$. En el estudio, solo en el 32 \% la mascarilla les cubría boca y nariz con buen acople y, al margen de cómo lo usaban, el 50 \% estaba en condiciones óptimas, sin rasgaduras ni rotas.

Akhtar y un grupo de investigadores observaron que mantener el distanciamiento no es factible en situaciones sociales e interacciones médicas cotidianas. Se basan en el hecho de que con un distanciamiento social comprometido, las gotas de tos y estornudos en el aire pueden atravesar todas las mascarillas probadas (excepto la N95), incluso cuando se asume que están totalmente ceñidas al rostro. Un solo estornudo puede contener de 10 a $200 \times 106$ partículas de virus, de acuerdo a la carga viral del portador ${ }^{(13)}$. Según el tipo, las mascarillas parecen ser eficaces solo cuando la persona susceptible está expuesta a concentraciones de virus menores que $5 \times 106$ (estornudo o tos), para las máscaras quirúrgicas y de tela probadas en el estudio ${ }^{(14)}$. Chanjuan Sun y Zhigiang Zhai demostraron que mantener un distanciamiento social adecuado es muy importante en la prevención del contagio por el SARS-CoV-2. Ellos probaron que la distancia mínima segura para realizar actividades sociales regulares, que impliquen hablar y respirar, es de 1,60 a 3 metros, mientras que la máxima distancia de transmisión podría llegar hasta los 8,2 metros con una posibilidad de contagio del $5 \%$, lo que también depende del ambiente en el que se encuentren y 
Factores sociodemográficos relacionados con el uso adecuado de las mascarillas y el distanciamiento social apropiado para evitar el contagio del SARS-CoV-2 en un mercado de abastos en Lima, Perú

la ventilación del lugar en el que se realice dicha actividad social (15). En nuestra investigación encontramos que el $94,00 \%$ de los participantes mantenían una distancia menor de 1,50 metros, y cuando se les consultó cuál era el distanciamiento social adecuado, el 75,80 \% consideró que era mayor de 1,50 m. Esta opinión fue incongruente con la evidencia práctica.

En una revisión sistemática sobre el distanciamiento social, el uso de mascarillas y protección ocular para prevenir la transmisión de persona a persona del SARS-CoV-2, Chu et al. concluyeron que una distancia física de 1 metro, al menos, está asociada con una gran reducción en la transmisión del virus y que distancias mayores de 2 metros podrían ser aún más eficaces en la prevención de la transmisión. La información previa sugiere que el uso de las mascarillas protege a las personas contra la infección y que la protección ocular podría dar un beneficio adicional (16).

El mercado de abastos de Surquillo, considerado un centro formal, mantiene ciertos regímenes de control como aforo limitado, toma de temperatura a los visitantes, uso de mascarilla al ingreso, alcohol en gel para las manos y pruebas diagnósticas para SARS-CoV-2 periódicas a los trabajadores. Estas medidas surgieron de intervenciones realizadas como parte del plan multisectorial \#TeCuidoPerú del Ministerio de Salud. En el Mercado Mayorista de Santa Anita, de 190 pruebas rápidas realizadas a trabajadores, 132 resultaron positivas para COVID-19 ${ }^{(17)}$. Las disposiciones del Gobierno deben ser cumplidas por los comerciantes y por las personas que acuden a lugares públicos. En este estudio, solo el $27,80 \%$ de los visitantes cumplió con el uso adecuado de las mascarillas, mientras que el $94,00 \%$ no cumplió con el distanciamiento social. Esta situación debe ser crítica en mercados informales, donde no se controlan las disposiciones de salud.

En esta investigación, el 8,80\% de los participantes menciona que resultó positivo para alguna prueba diagnóstica para la COVID-19, y el 17,90\% refirió que alguno de sus familiares en el entorno inmediato tuvo también una respuesta positiva, lo cual aumenta el riesgo de contagio si no se cumplen las medidas de protección adecuadas y, al mismo tiempo, convertirse en vectores para nuevos contagios.

En un estudio sobre la transmisión e infección de SARS-CoV-2 en un campamento de EE. UU., en el que las habitaciones eran compartidas hasta por 15 personas, se observó que, a pesar de haber aplicado las medidas establecidas por el CDC, se contagió el $44 \%$ de un total de 597 participantes ${ }^{(18)}$.

La OMS y varios países han recomendado el uso de las mascarillas caseras en público en áreas en las que podría ocurrir una transmisión comunitaria. Si bien efectividad de esta medida es limitada, ya que solo ofrece un $5 \%$ de protección y contención, puede lograr una reducción significativa de los contagios y, por lo tanto, de las muertes, en un porcentaje del 3 al $5 \%$ según Worby et al. ${ }^{(19)}$. Mitzeetal. emplearon datos de los contagios de las diferentes regiones en Alemania (donde las directivas del uso obligatorio de las mascarillas se dieron en diferentes periodos en las distintas regiones) y sus resultados mostraron que tan solo 20 días después de que el uso de mascarillas fuera obligatorio, la cantidad de nuevos infectados se redujo en $45 \%$, aproximadamente ${ }^{(20)}$. Asimismo, en Tailandia, un grupo de investigadores encontró evidencias de que el uso de mascarillas, el lavado de manos y el distanciamiento social están independientemente asociados a la disminución del contagio del SARS-CoV-2 en el entorno comunitario (21).

El sesgo de observación se consideró como una limitación en este estudio; para disminuirlo, los encuestadores fueron capacitados previamente. Para disminuir el sesgo de medición se utilizó una vara rígida que tenía una cinta métrica adosada. Se concluye que el grado de instrucción tiene relación significativa con el uso adecuado de las mascarillas: el 27,80 \% las empleó de manera adecuada, criterio basado en el cumplimiento de tres condiciones: el tipo correcto, la buena condición de la mascarilla y si cubría adecuadamente boca y nariz con buen acople. El $94 \%$ mantuvo una distancia menor de 1,50 metros.

Con base en la evidencia científica publicada, que indica que el uso de las mascarillas y el distanciamiento social funcionan como mecanismos importantes de protección, y para hacer efectivasestas intervenciones no farmacológicas, es necesario realizar campañas de información en los medios de comunicación y distribuir mascarillas, sobre todo, a personas de escasos recursos ${ }^{(22)}$. Esta investigación de tipo comunitaria refleja la situación de un mercado de abastos formal, localizado en un sector de clase media, en un momento en el cual no se cuenta con la vacuna para el SARS-CoV-2. La situación en los mercados informales debe ser crítica, por lo que se recomienda realizar estudios en estos lugares para establecer recomendaciones y medidas de control.

Contribuciones de los autores: RHC y NE han participado en la concepción y diseño del artículo. RHC se encargó de la recolección de los datos y análisis estadístico de datos. Todos los autores participaron en la interpretación de los datos, redacción del manuscrito, revisión crítica del manuscrito y aprobaron la versión final.

Fuente de financiamiento: El artículo fue financiado por los autores.

Conflictos de interés: Este estudio forma parte de la tesis que será sustentada por Renzo Hurtado Cuba. La Dra. Espíritu ha sido asesora de dicha tesis. 


\section{REFERENCIAS BIBLIOGRÁFICAS}

1. Lau JTF, Tsui H, Lau M, Yang X. SARS transmission, risk factors, and prevention in Hong Kong. Emerg Infect Dis. 2004; 10(4): 587-92.

2. Cheng VC-C, Wong S-C, Chuang VW-M, So SY-C, Chen JH-K, Sridhar S, et al. The role of community-wide wearing of face mask for control of coronavirus disease 2019 (COVID-19) epidemic due to SARS-CoV-2. J Infect. 2020; 81(1): 107-14.

3. Fischer EP, Fischer MC, Grass D, Henrion I, Warren WS, Westman E. Low-cost measurement of face mask efficacy for filtering expelled droplets during speech. Sci Adv. 2020; 6(36): eabd3083.

4. World Health Organization. Weekly epidemiological update on COVID-19 - 1 June 2021 [Internet]. WHO; 2021. Disponible en: https: // www. who.int/publications/m/item/weekly-epidemiologicalupdate-on-covid-19---1-june-2021

5. Ministerio de Salud. Coronavirus (COVID-19) en el Perú [Internet]. MINSA: Perú; 2020. Disponible en: https://www.gob.pe/coronavirus

6. AFP. Perú registra la tasa de muertes por Covid-19 más alta del mundo tras actualización de fallecidos [Internet]. Diario El Economista: Perú; 2021. Disponible en: https://www. eleconomista.com.mx/internacionales/Peru-registra-la-tasa-demuertes-por-Covid-19-mas-alta-del-mundo-tras-actualizacion-defallecidos-20210531-0070.html

7. AFP Interactive. Evolución de la cantidad de casos y muertos por Covid-19 [Internet]. 2021. Disponible en: https://interactive.afp. $\mathrm{com} /$ graphics/Evolucion-de-la-cantidad-de-casos-y-muertos-porCovid-19_603

8. Ministerio de Salud. Campaña Nacional de Vacunación contra la COVID-19 [Internet]. MINSA: Perú; 2021. Disponible en: https:// www.gob.pe/pongoelhombro

9. World Health Organization. Advice on the use of masks in the community, during home care and in healthcare settings in the context of the novel coronavirus (COVID-19) outbreak [Internet]. WHO; 2020. Disponible en: https://www.who.int/publicationsdetail/advice-on-the-use-of-masks-in-the-community-duringhome-care-and-in-healthcare-settings-in-the-context-of-the-novelcoronavirus-(2019-ncov)-outbreak

10. Greenhalgh T, Schmid MB, Czypionka T, Bassler D, Gruer L. Face masks for the public during the covid-19 crisis. BMJ. 2020; 369: $\mathrm{m} 1435$.

11. Gandhi M, Rutherford GW. Facial masking for Covid-19 - Potential for "variolation" as we await a vaccine. N Engl J Med. 2020; 383: e101.

12. Li T, Liu Y, Li M, Qian X, Dai SY. Mask or no mask for COVID-19: A public health and market study. PLoS One. 2020; 15(8): e0237691.

13. Schijven J, Vermeulen LC, Swart A, Meijer A, Duizer E, de Roda Husman AM. Exposure assessment for airborne transmission of SARSCoV-2 via breathing, speaking, coughing and sneezing. medRxiv. 2020.

14. Akhtar J, Garcia AL, Saenz L, Kuravi S, Shu F, Kota K. Can face masks offer protection from airborne sneeze and cough droplets in closeup, face-to-face human interactions? - A quantitative study. Phys Fluids (1994). 2020; 32(12): 127112.

15. Sun $C$, Zhai $Z$. The efficacy of social distance and ventilation effectiveness in preventing COVID-19 transmission. Sustain Cities Soc. 2020; 62: 102390.

16. Chu DK, Akl EA, Duda S, Solo K, Yaacoub S, Schünemann HJ. Physical distancing, face masks, and eye protection to prevent person-toperson transmission of SARS-CoV-2 and COVID-19: a systematic review and meta-analysis. Lancet. 2020; 395(10242): 1973-87.

17. Ministerio de Salud. Grupo "Te Cuido Perú" continúa realizando operativos en mercados para detectar casos positivos de COVID-19 [Internet]. MINSA: Perú; 2020. Disponible en: https://www.gob.pe/ institucion/mindef/noticias/143640-grupo-te-cuido-peru-continua- realizando-operativos-en-mercados-para-detectar-casos-positivosde-covid-19

18. Szablewski CM, Chang KT, Brown MM, Chu VT, Yousaf AR, Anyalechi N, et al. SARS-CoV-2 Transmission and Infection Among Attendees of an Overnight Camp - Georgia, June 2020. MMWR Morb Mortal Wkly Rep. 2020; 69(31): 1023-5.

19. Worby $\mathrm{CJ}$, Chang H-H. Face mask use in the general population and optimal resource allocation during the COVID-19 pandemic. Nat Commun. 2020; 11: 4049.

20. Mitze T, Kosfeld R, Rode J, Wälde K. Face masks considerably reduce COVID-19 cases in Germany. Proc Natl Acad Sci. 2020; 117(51): 32293-301.

21. Doung-Ngern $P$, Suphanchaimat $R$, Panjangampatthana $A$, Janekrongtham C, Ruampoom D, Daochaeng N, et al. Case-control study of use of personal protective measures and risk for SARS-CoV 2 infection, Thailand. Emerg Infect Dis. 2020; 26(11): 2607-16.

22. Bo Y, Guo C, Lin C, Zeng Y, Li HB, Zhang Y, et al. Effectiveness of non-pharmaceutical interventions on COVID-19 transmission in 190 countries from 23 January to 13 April 2020. Int J Infect Dis. 2021; 102: 247-53.

\section{Correspondencia:}

Renzo Hurtado Cuba

Dirección: Los Ingenieros 665 dpto. 501. Valle Hermoso Surco. Lima, Perú.

Teléfono: +51 969439161

Correo electrónico: rhurtadocuba@gmail.com

Recibido: 12 de febrero de 2021 Evaluado: 02 de junio de 2021 Aprobado: 09 de junio de 2021

๑ La revista. Publicado por Universidad de San Martín de Porres, Perú. (cc) Br Licencia de Creative Commons Artículo en acceso abierto bajo términos de Licencia Creative Commons Atribución 4.0 Internacional. (http://creativecommons.org/licenses/by/4.0/)

ORCID iDs

Renzo Hurtado-Cuba $\odot$ https://orcid.org/0000-0002-0904-5892

Nora Espíritu $\quad$ https://orcid.org/0000-0002-6340-0285 